SERKOWSKI: The assumption that there is a little magnetic field which is aligning the grains has only one advantage, namely that it is easy to explain very high polarization (which is observed in red stars). But on the other hand there are many difficulties: First it is very common that the position angle depends strongly on wave length. This seems hard to explain if the polarization is produced only along the path from the star to us by aligned grains. There also must be large polarization produced by the simple reflection nebula mechanism without any alignement. If the grains are very small then there should be nearly 100 percent polarization for a scattering angle of 90 degrees. Therefore the assumption of a magnetic field is not necessary for such stars. The difficulties in explaining the large polarization for some objects disappear if we only assume that the star itself is almost completely obstructed and that we see practically only scattered light.

\title{
To the Explanation of the Intrinsic Polarization of the Light of Some Red Long Period Variables
}

\author{
CH. FRIEDEMANN (Jena)
}

(The paper was presented at the Second IAU Colloquium on Interstellar Dust held at Jena 1969)

Summary: The time dependent polarization of the light of some red long period variables can be explained using the simple model of the oblique rotator. Near the magnetic poles the dust particles in the outer layers of the stellar atmosphere could be aligned in the magnetic field by the DAVIS-GREENSTEIN mechanism. For the stars VCVn, $\mathrm{L}_{2}$ Pup, R Leo and R Peg the spatial orientation of the rotational axis, the angle between the magnetic poles and the rotational axis and the period of the revolution of the magnetic poles around the rotational axis were derived from the observational material. The proposed hypothesis gives an explanation of the essential observational facts concerning the intrinsic polarization of some of the red variables.

\section{To the Explanation of the Polarization of the Radiation of Mu Cephei}

\author{
CH. FRIEDEMANN (Iena)
}

Assuming a stellar magnetic dipole-field which aligns particles in the stellar atmosphere the observed polarization of the light of $\mu$ Cephei can be explained. The spatial orientation of the rotational axis, the angle between the magnetic axis and the rotational axis and the period of rotation were derived from the observational values. The period of rotation amounts to about $1650^{\mathrm{d}} \pm 10^{\mathrm{d}}$. In case aligned graphite flakes are present in the outer layers of the stellar atmosphere the calculated degree of polarization amounts to about $1.5 \%$. According to the chemical composition of the atmosphere it is more probably that siliciumcarbide particles cause the polarization. In this case the degree of polarization amounts to approximately $0.75 \%$. The portion of polarization originating in interstellar space was estimated to $p_{\mathrm{i}} \approx 0.8 \%$ and the position angle is about $\Theta_{\mathrm{i}} \approx 45^{\circ}$.

Both papers will be published in the "Astronomische Nachrichten". 